\title{
Role of nanostructure on the optical waveguiding properties of epitaxial $\mathrm{LiNbO}_{3}$ films
}

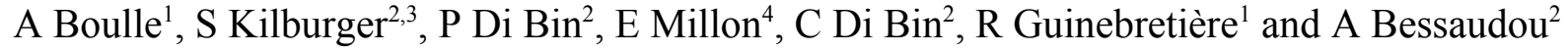 \\ ${ }^{1}$ Laboratoire Science des Procédés Céramiques et de Traitements de Surfaces (SPCTS), \\ CNRS UMR 6638, ENSCI, 47 avenue Albert Thomas, 87065 Limoges - France \\ ${ }^{2}$ Laboratoire XLIM, CNRS UMR 6172, 123 avenue Albert Thomas, 87060 Limoges - France \\ ${ }^{3}$ Laboratoire LSMCL, Université de Metz, 1 bvd Arago, 57078 Metz Cedex3 - France \\ ${ }^{4}$ Laboratoire GREMI, Université d'Orléans, CNRS UMR 6606, 14 rue d'Issoudun, BP 6744, \\ 45067 Orléans - France
}

Corresponding author : alexandre.boulle@unilim.fr

\begin{abstract}
High quality $\mathrm{LiNbO}_{3}(\mathrm{LN})$ epitaxial films have been grown onto (001) $\mathrm{Al}_{2} \mathrm{O}_{3}$ substrates by pulsed laser deposition. The deposition conditions have been optimized using experimental designs to promote the growth of highly (001)-oriented, phase pure and light-guiding LN films. The structural and nanostructural properties have been investigated by X-ray diffraction reciprocal space mapping and the guiding properties have been investigated by $\mathrm{m}$-line spectroscopy and measurements of the light propagation losses. In particular it is shown that the film composition, the state of strain, the film thickness, the film roughness, the lateral extension of the crystallites building up the film as well as the mosaicity can be determined by a careful examination of the X-ray reciprocal space maps associated with simulation of the diffracted intensity distribution in reciprocal space. The guiding properties have been correlated with the nanostructural properties of the films: whereas light guiding has been clearly observed in single crystal - like films, the existence of a mosaic structure, made of nanocrystalline domains, is shown to be detrimental to the guiding properties.
\end{abstract}




\section{Introduction}

Lithium niobate $\left(\mathrm{LiNbO}_{3}: \mathrm{LN}\right)$ is a widely used optical material due to its interesting electro-optical and non-linear optical properties. LN is particularly used in optical devices such as waveguides, electro-optical modulators and frequency conversion (periodically poled lithium niobate) devices [1]. These applications are currently developed using bulk materials but thin films offer several potential advantages. For example, in a vertically-designed electro-optical modulator structure including bottom and top electrodes parallel to the LN film, the low film thickness allows a much lower inter-electrodes distance than usual coplanar electrodes used in bulk LN technology. Furthermore, a better matching between the optical polarization and the applied electric field improves the overlap integral between these both fields. This leads to a decrease of the drive voltages, and allows integration and device size reduction.

In the past few years, lithium niobate films have been prepared using a large variety of techniques, namely sol-gel [2], sputtering [3], liquid phase epitaxy [4], metalorganic chemical vapour deposition [5] and pulsed laser deposition (PLD) [6-8]. Among these techniques, PLD appears to be one of the most promising because of its ability to reproduce the composition of the target in the film. The most important issue is to succeed in the deposition of high quality LN films with reduced optical losses.

It has often been argued that the structural quality of the films has a huge influence on the optical properties, especially on the optical losses $[5,9,10]$. Our work has therefore consisted in optimizing the deposition parameters from the experimental PLD set-up to produce high quality lithium niobate films epitaxially grown on (001) sapphire. Several tens of films have been grown and characterized using X-ray diffraction (XRD) and optical measurements (section 2). XRD reciprocal space mapping has been used to characterize the structural and nanostructural properties of the LN films (section 3). In particular, a careful analysis of asymmetrical (018) and symmetrical (00l) reciprocal spaces maps (RSMs) allows derivation in a non-destructive manner of the film composition (this point is especially important for these materials where the volatility of $\mathrm{Li}$ is a well-known issue [11-13]), the amount of strain, the film thickness and roughness, the lateral crystallite size and the mosaicity. The optical properties have been evaluated by excitation of guided modes in the films with a prism coupler. The light guiding characteristics at $\lambda=633 \mathrm{~nm}$ are presented (section 4). Finally, we show that light guiding is favoured in single crystal - like films, 
whereas the existence of a mosaic structure, made of nanocrystalline domains, is detrimental to the guiding properties (section 5).

\section{Experimental}

\subsection{Film growth}

The LN films have been grown in an ultra-high vacuum stainless steel vessel evacuated by a turbomolecular pump to a residual pressure of $10^{-6}$ mbar. An ArF excimer laser (Lambda Physik Compex 201) operating in the UV range at $193 \mathrm{~nm}$ wavelength, at a repetition rate of $5 \mathrm{~Hz}$, with a pulse duration of about $23 \mathrm{~ns}$, is used for the PLD process. In order to limit droplets and particle ejection during the deposition, a (congruent) LN singlecrystal has been used as the target material. The target is mounted on a rotating holder to ensure a uniform wear and the laser beam incidence is $45^{\circ}$ with respect to the target normal. The films were grown on sapphire (001) substrates $\left(10 \times 10 \mathrm{~mm}^{2}\right)$. High quality sapphire substrates are commercially available and both materials exhibit a similar structure (although the lattice mismatch is quite high, see below). The refractive indices of sapphire $\left(n_{e}=1.7575\right.$; $\mathrm{n}_{\mathrm{o}}=1.7654$ at $\left.\lambda=633 \mathrm{~nm}\right)$ are lower than those of $\operatorname{LN}\left(\mathrm{n}_{\mathrm{e}}=2.2027 ; \mathrm{n}_{\mathrm{o}}=2.2863\right.$ at $\left.\lambda=633 \mathrm{~nm}\right)$, which is a necessary condition for the optical confinement in waveguides. For these reasons, sapphire is the most widely used substrate material for waveguiding applications [3, 5-16]. However, it must be emphasized that because of this rather large refractive index contrast ( 0.5) single mode waveguiding can only be observed in relatively thin films $(<150 \mathrm{~nm})$.

The experimental conditions for producing epitaxial and stoichiometric $\mathrm{LiNbO}_{3}$ thin films in general depend on the laser wavelength and the experimental set-up used. In the present study we used experimental designs to optimize the experimental deposition parameters (oxygen pressure, substrate temperature and laser fluence). In a first step, the pressure was varied between 0.1 and 0.3 mbar, the temperature was varied between 600 and $750^{\circ} \mathrm{C}$ and the laser fluence was varied between 0.5 and $0.9 \mathrm{~J} / \mathrm{cm}^{2}$. A screening design within these experimental ranges revealed that the critical parameters to grow single phase LN films are the oxygen pressure and the laser fluence. In a second step a principal components analysis was carried out to examine correlations between the experimental factors and the film properties. This analysis revealed that single phase LN films were obtained for increasing pressure and laser fluence, whereas the substrate temperature had no effect in the investigated 
range. In the following, the substrate temperature was set to $650^{\circ} \mathrm{C}$. Finally, the oxygen pressure and laser fluence have been optimized using a response surface methodology. This technique allows examination of the relationship between experimental parameters and specific response variables [17]. For that purpose, the pressure was varied between 0.3 and $1.5 \mathrm{mbar}$ and the laser fluence between 1.2 and $2 \mathrm{~J} / \mathrm{cm}^{2}$. The responses that were used to perform the analysis were (i) the presence of the secondary phases (i.e. other phases than pure $\mathrm{LiNbO}_{3}$ ), (ii) the quality of light propagation in the films and (iii) the density of droplets at the surface of the films. The best results were obtained for an oxygen pressure $P_{\mathrm{O} 2}=1.2 \mathrm{mbar}$ and a laser fluence $F=2 \mathrm{~J} / \mathrm{cm}^{2}$. The influence of the target to substrate distance has not been explored in this study and was set to $49 \mathrm{~mm}$. Prior to deposition, to avoid surface pollution and to ensure a homogeneous emission of LN species in the plasma produced by the laser ablation of the target, a pre-ablation was performed during $10 \mathrm{~min}$. The cooling step which follows the deposition was performed at the same oxygen pressure of 1.2 mbar for 2 hours. The deposited rate, evaluated by thickness measurements for a given deposition time, is close to $5 \mathrm{~nm} / \mathrm{min}$.

\subsection{X-ray diffraction}

The films were characterized by high-resolution XRD. A laboratory diffractometer with a rotating $\mathrm{Cu}$ anode, a four-reflection monochromator and a curved position sensitive detector were used to record RSMs. A five-movement sample holder allows precise sample positioning. The X-ray beam impinging on the sample is monochromatic $\left(\mathrm{Cu} \mathrm{K} \alpha_{1}, \Delta \lambda /\right.$ $\left.\lambda=1.4 \times 10^{-4}\right)$ and parallel in the detector plane $(\Delta \theta=12$ arcsec $)$ with dimensions $10 \times 0.09 \mathrm{~mm}^{2}$ so that a large volume of the sample is analyzed which provides statistically significant averaged values. A detailed description of the set-up has been given elsewhere [18-20]. A RSM represents the scattered intensity in a particular $\left(Q_{x}, Q_{z}\right)$ plane, where $Q_{x}$ and $Q_{z}$ are the components of the scattering vector $Q(Q=4 \pi \sin \theta / \lambda)$ in the film plane and perpendicular to it, respectively. In the following experiments, $Q_{x}$ and $Q_{z}$ have been set parallel to the [010]* and [001]* directions of sapphire and will henceforth be denoted $Q_{[010]}$ and $Q_{[001]}$, respectively. In the previous notation, the * simply indicates that directions considered are reciprocal lattice directions, corresponding to the normal to the (010) and (001) planes, respectively. 
Asymmetrical (018) RSMs were used to determine the amount of strain stored in the film together with the bulk (i.e. strain-free) lattice parameters of LN. These latter parameters permit determination of the film composition. Symmetrical (006) and (0012) RSMs were used to determine the film thickness and thickness fluctuations (i.e. roughness), the lateral crystallite size, the mosaicity and the amount of heterogeneous strain.

\subsection{Optical properties}

The optical waveguiding properties of the film have been investigated using the prism coupling method, also called m-line spectroscopy, with a $633 \mathrm{~nm}$ He-Ne laser [21]. For this purpose, the film under investigation is pressed against the base of a rutile coupling prism (figure 1). The laser beam, linearly polarized (TE or TM) is focused by a lens into the prism so that the coupling spot coincides with the prism base close to the prism apex (less than 1 $\mathrm{mm})$. The prism and the film sit on an $\mathrm{x}-\mathrm{y}$ translation stage that is mounted on a precision goniometer. The light is totally reflected at the prism base, except when the incoming light is coupled into the film and propagates. This is achieved when the phase-matching condition is realized between the beam and the excited guided mode of the waveguide. Such a coupling creates a dark line (figure 1) in the reflected beam indicating that the corresponding optical power is coupled into the waveguide. This condition is realized by varying the angle between the incident beam and the prism base. The knowledge of this angle gives an estimation of the thickness of the film (assuming the refractive indices are equal to those of a bulk $\mathrm{LiNbO}_{3}$ single crystal).

The optical guiding properties of the film are mainly characterized by the evaluation of the propagation losses. The method used is the classical image analysis of the scattered optical power through the $\mathrm{LiNbO}_{3}$-air interface along the propagation axis of the film. A fitted spatial averaging of the image reduces the influence of bright spots on the surface caused by microscopic dust particles and surface defects that may reduce the precision of the loss measurements. The evolution of the power measured along the guided streak is calculated and expressed in $\mathrm{dB} / \mathrm{cm}$ by computing the mean slope of the evolution of the optical power expressed in the logarithmic unit $(\mathrm{dBm})$ as a function of propagation distance. The set-up allows propagation and measurements up to $9 \mathrm{~mm}$. 


\section{Structural and nanostructural characterization}

$\mathrm{LiNbO}_{3}$ and $\mathrm{Al}_{2} \mathrm{O}_{3}$ exhibit a trigonal symmetry and have a similar structure based on a hexagonal close-packed oxygen sublattice where $2 / 3$ of the octahedral sites are occupied by the cations. Along the $\mathrm{c}$ axis the cation stacking is $\mathrm{Al}^{3+} / \mathrm{Al}^{3+} /$ (vacancy) in sapphire and $\mathrm{Li}^{+} / \mathrm{Nb}^{5+} /$ (vacancy) in LN (LN being a ferroelectric material, this stacking is observed when moving along the $+\mathrm{c}$ polar direction). $\mathrm{LiNbO}_{3}$ has space group $\mathrm{R} 3 \mathrm{c}$ and hexagonal lattice parameters $\mathrm{a}=5.1483 \AA$ and $\mathrm{c}=13.863 \AA . \mathrm{Al}_{2} \mathrm{O}_{3}$ has space group $\mathrm{R}-3 \mathrm{c}$ and hexagonal lattice parameters $\mathrm{a}=4.7597 \AA$ and $\mathrm{c}=12.993 \AA$. At room temperature the misfit strain is extremely high, $-7.5 \%$ (compressive). Taking into account the coefficient of thermal expansion (along the direction a) of $\mathrm{LiNbO}_{3}\left(15 \times 10^{-6} \mathrm{~K}^{-1}\right)$ and $\mathrm{Al}_{2} \mathrm{O}_{3}\left(5.6 \times 10^{-6} \mathrm{~K}^{-1}\right)$, the misfit strain at the deposition temperature is increased to $-8.1 \%$. It is obviously unlikely that the film could sustain such a high level of strain.

It is now broadly accepted that films exhibiting a relatively large misfit ( $>5 \%)$ grow in a strain-relaxed state where the strain energy associated with the misfit is considerably lowered by the formation of domains in which integral multiples of the lattice constants match (or nearly match) across the interface [22,23], e.g. n lattice planes of the film nearly match $\mathrm{n} \pm 1$ lattice planes of the substrate (the so-called domain-matching epitaxy). In this case each domain contains a "geometrical" misfit dislocation: there is a missing lattice plane (in the case of $n / n+1$ matching) or an additional plane (in the case of $n / n-1$ matching) in the film. The overall strain energy then depends on the residual misfit (in the case where the matching is not perfect, which is almost always the case), and the energy associated with the dislocation [24]. The geometrical dislocation is directly generated at the interface during growth. This is in contrast with conventional misfit dislocations that are encountered in small misfit systems. Conventional misfit dislocations are generated at the film surface and glide to the interface during growth under the action of the epitaxial stress. Since no glide is involved, the Burgers vector of the geometrical dislocation does not necessarily belong to an active slip system of the material [22]. Moreover, there are no threading segments associated with these dislocations so that the strain is confined close to the interface and high quality films can therefore be grown [23]. The results obtained for the $\mathrm{LN} / \mathrm{Al}_{2} \mathrm{O}_{3}$ system will be discussed in the light of this concept. 


\subsection{Film orientation}

RSMs recorded close to the (006) and (018) reflections of $\mathrm{Al}_{2} \mathrm{O}_{3}$ and $\mathrm{LiNbO}_{3}$ are shown in figure 2. The inclined streak running through the reflections of the film and the substrate is due to the transmittance function of the detector [19]. The reflections of the film are streaked along the $[001]^{*}$ direction which originates from the finite film thickness. They are also broadened in the direction perpendicular to the reciprocal lattice vector of the reflections considered (i.e. perpendicular to $\mathrm{Q}_{[001]}$ for the (006) reflection and perpendicular to $\mathrm{Q}_{[018]}$ for the (018) reflection) which is indicative of a disorder that is rotational in nature, i.e. mosaicity [25]. These features will be discussed in detail in the next section.

The alignment of the (006) reciprocal lattice points along the $[001]^{*}$ direction (figure 2a) confirms the out-of-plane orientation: $(001)_{\mathrm{LN}} \|(001)_{\mathrm{Al} 203}$. The presence of both $(018)$ reflections from the film and the substrate in the plane defined by the directions $[010]^{*}$ and $[001]^{*}$ further indicates the following in-plane orientation: $[010]_{\mathrm{LN}}^{*} \|[010]_{\mathrm{Al2} O 3}^{*}$, which is equivalent to $[100]_{\mathrm{LN}} \|[100]_{\mathrm{Al} 203}$. The occurrence of an additional in-plane epitaxial variant, rotated by $60^{\circ}$ from the previous orientation (i.e. $[110]_{\mathrm{LN}}||[100]_{\mathrm{A} 1203}$ ), has been frequently mentioned in the literature $[5,8,9,14,15]$. In order to check the existence of this variant, we recorded a $\phi$-scan from the (018) reflection of $\mathrm{LiNbO}_{3}$ (figure 3). The $\phi$-scan indeed reveals the existence of a very weak peak corresponding to the $60^{\circ}$ variant. Its volume fraction can be estimated from the integrated intensities of the main peak $\left(I_{0}\right)$ and the peak corresponding to the variant $\left(I_{60}\right)$ as follows : $I_{0} /\left(I_{0}+I_{60}\right)$. It finally turns out that the volume fraction of the variant is only $6 \%$. The existence of this variant is due to the fact that for both orientations, the structure of $\mathrm{LiNbO}_{3}$ only differs in the cationic stacking sequence (i.e. the oxygen sublattice is identical). For the main orientation the stacking sequence at the interface is $\mathrm{Al}^{3+}$ / $\mathrm{Al}^{3+} /\left(\right.$ vacancy) $/ / \mathrm{Li}^{+} / \mathrm{Nb}^{5+}$ (inset (a) figure 3), whereas for the variant, the stacking sequence is $\mathrm{Al}^{3+} / \mathrm{Al}^{3+} / / \mathrm{Li}^{+} /\left(\right.$vacancy)$/ \mathrm{Nb}^{5+}$ (inset (b) figure 3). As mentioned in Ref. 9, although the difference between the interfacial energies is probably very weak, the main orientation is favoured because it preserves the cationic stacking sequence of the type cation/cation/ (vacancy) occurring in the substrate. This effect is more pronounced at temperatures where the mobility of Li is kept low [9]. Since the growth has been performed at a relatively low temperature $\left(650^{\circ} \mathrm{C}\right)$, it can be expected that the mobility of $\mathrm{Li}$ at this temperature is sufficient to favour the main orientation. This result is in good agreement with the work of Lee et al. [5] 
where $650^{\circ} \mathrm{C}$ was found to be the optimum temperature to prevent the formation of the variant. It is worth noting that no Li-deficient secondary phases $\left(e . g\right.$. $\left.\mathrm{LiNb}_{3} \mathrm{O}_{8}\right)$ are observed in the RSMs as a result of the optimized growth conditions described earlier, i.e. the films are grown with the correct stoichiometry (this point is discussed in detail below) without using any Li-enriched target and without using any in-situ or ex-situ annealing procedure.

\subsection{Composition and strain}

Since the film and the substrate exhibit the same oxygen sublattice (but a different cationic sublattice), the concept of domain-matching epitaxy is better understood when considering oxygen planes instead of lattice planes. Taking into account the previously determined film/substrate orientations, a near coincidence site lattice (NCSL) consisting of 12 oxygen planes of LN matching 13 oxygen planes of sapphire can be defined at the growth temperature. With this NCSL, the residual misfit strain, defined as $\left(13 d_{\mathrm{OO}, \mathrm{s}}-12 d_{\mathrm{OO}, \mathrm{f}}\right) / 12 d_{\mathrm{OO}, \mathrm{f}}$, is reduced to $0.26 \%$, where $d_{\mathrm{OO}}$ is the distance between the oxygen planes and the subscripts 's' and 'f' stand for 'substrate' and 'film', respectively. The remaining strain can be relieved either by the formation of conventional misfit dislocations or by local variations of the size of the coincidence domains [22,23]. Each domain, consisting of 12 oxygen planes, contains a geometrical misfit dislocation consisting of a missing oxygen plane in LN, with Burgers vector $d_{\mathrm{OO}}(5.1962 \AA$ at the growth temperature, $5.1476 \AA$ at room temperature). The mean distance between such dislocations is hence $12 d_{\mathrm{oO}}$, that is $6.2 \mathrm{~nm}$, which is in good agreement with the values obtained by transmission electron microscopy in the same system $[9,16,26]$. These dislocations are periodically distributed at the interface, and therefore they do not generate heterogeneous strain in the film volume [27] (i.e. the heterogeneous strain is confined close to the interface) which hence allows growing high quality epitaxial LN films, despite the high value of the lattice misfit.

In order to confirm this mechanism we now investigate the state of strain of the LN films. In principle, the state of strain can be obtained using the measured $c$ parameter of LN, obtained from the position of the (006) peak: $e_{z z}=\left(c-c_{b}\right) / c_{b}$, where $c_{b}$ is the bulk (strain-free) $c$ lattice parameter of LN. The in-plane strain then follows from $e_{x x}=-v_{2} e_{z z}$, where $v_{2}$ is the biaxial Poisson's ratio. However, because of the well-known aforementioned issues of $\mathrm{Li}$ volatility, the exact value of $c_{b}$ is probably not accurately known, so that simply setting $c_{b}$ to 
its value corresponding to the composition of the target (i.e. congruent) might be an unwary assumption. To circumvent this problem we make use of the asymmetrical RSMs [28,29] (figure $2 \mathrm{~b}$ ). The coordinates of the film reflection along the $[010]^{*}$ and $[001]^{*}$ directions allow derivation of the $b$ and $c$ parameters, from which the strain-free lattice parameter $a_{b}$ can be derived (since in the hexagonal geometry $a=b$ ):

$$
a_{b}=\frac{c+v_{2} k_{b} a}{1+v_{2}}
$$

$k_{b}$ is the $c_{b} / a_{b}$ ratio which is only weakly dependent on the composition. Indeed, for a molar fraction of $\mathrm{Li}, x_{\mathrm{Li}}$, ranging between $45 \%$ and $50 \%, k_{b}$ ranges from 2.6934 to 2.6917 [30]. The calculations were performed with different values of $k_{b}$, and the results were identical within the experimental uncertainty $\left(\sim 10^{-4} \AA\right)$. In the following we shall therefore use the value corresponding to the stoichiometric composition $\left(x_{\mathrm{Li}}=50 \%\right)$. Finally, for the $(001)$ orientation of a hexagonal material, the biaxial Poisson's ratio is $v_{2}=2 \mathrm{C}_{13} / \mathrm{C}_{33}$ [31] (where $\mathrm{C}_{\mathrm{ij}}$ are the elastic stiffness coefficients of the material); in the case of LN this yields 0.62 . For the LN film presented here we obtain $a_{b}=5.1497 \AA$ which is slightly greater than the value corresponding to the stoichiometric composition. The influence of the composition on the lattice parameters of LN has been studied by Malovichko et al. [30]. They have shown that in the range $x_{\mathrm{Li}}=45-50 \%$ the $a$ lattice parameter of $\mathrm{LN}$ can be written $: a=5.2276-0.16 x_{\mathrm{Li}}$. In the present case we obtain $x_{\mathrm{Li}}=48.7 \%$ which is very close to the congruent composition (48.4 $-48.5 \%$ ) [30]. Since we have used a congruent single crystalline target which is ablated in oxygen atmosphere under controlled pressure, this result proves unambiguously that the LN species are stoichiometrically transferred from the target to the substrate without loss of Li.

The in-plane strain can then be computed according to $e_{x x}=\left(a-a_{b}\right) / a_{b}$ and we obtain $e_{x x}=0.59 \%$ (tensile). At first sight this result might be surprising since $a_{\mathrm{LN}}>a_{\mathrm{Al} 203}$, one would expect compressive strain. However, this can be understood by considering the differences in the coefficients of thermal expansion (CTE) of $\mathrm{LN}$ and $\mathrm{Al}_{2} \mathrm{O}_{3}$. The in-plane CTE of $\mathrm{LN}$ is $\alpha_{a, \mathrm{f}}=15 \times 10^{-6} \mathrm{~K}^{-1}$, whereas the in-plane CTE of sapphire is $\alpha_{a, \mathrm{~s}}=5.6 \times 10^{-6} \mathrm{~K}^{-1}$. When cooling from the growth temperature, $T_{\mathrm{g}}$, down to the measurement temperature, $T_{\mathrm{m}}$, the film is hence under biaxial tensile strain such as $e_{\mathrm{th}}=\left(\alpha_{a, \mathrm{f}}-\alpha_{a, \mathrm{~s}}\right)\left(T_{\mathrm{g}}-T_{\mathrm{m}}\right)$. We here obtain $\mathrm{e}_{t h}=0.59 \%$ which exactly equals the measured in-plane strain. This confirms the domain-matching epitaxial growth suggested earlier: the film grows at $650^{\circ} \mathrm{C}$ in a fully strain-relaxed state $\left(e_{x x} \approx 0\right)$ whereas thermal strain is stored in the film upon cooling down. The equality $e_{x x}=\mathrm{e}_{t h}$ also 
indicates that the film should be free of nano- and micro-cracks which are known to be the main mechanism relieving the thermal strain energy [15]. This is indeed confirmed by optical microscopy observations of the surface of the film where no cracking was observed [32]. In the following section we investigate the structural quality of the film.

\subsection{Nanostructure and defects}

The analysis of the intensity distribution contained in the reciprocal lattice points of the film can provide a wealth of information concerning the film micro- and nanostructure. In particular, considering the symmetrical $(00 l)$ reflections, scans extracted along the [001] $]^{*}$ direction ( $Q_{z}$-scans) are sensitive to the film thickness, thickness fluctuation (i.e. roughness) and the $z z$ components of the homogeneous and heterogeneous strain tensor, whereas scans extracted along the $[010]^{*}$ direction $\left(Q_{x}\right.$-scans) are sensitive to the lateral extension of the crystallites and the $x z$ component of the heterogeneous strain tensor (i.e. mosaicity) [33]. Experimental $Q_{z^{-}}$and $Q_{x}$-scans extracted from (006) and (00 12) RSMs, together with calculated curves are displayed in figure 4 . We first consider the $Q_{z}$-scans.

For a symmetrical reflection with reciprocal lattice vector $\left(0,0, h_{z}\right)$, the intensity distribution in the direction normal to the interface is given by [33]

$$
I\left(q_{z}\right)=\int d z \cdot \tilde{R}(z) V(z) G(z) \exp \left(i q_{z} z\right)
$$

where $q_{z}$ is the deviation (in the $z$ direction) of the scattering vector $\mathbf{Q}$ from the Bragg position $\left(q_{z}=Q_{z}-h_{z}\right) . V(z)$ is the correlation volume which depends on the average film thickness, $<t>$, and the root-mean-squared (rms) roughness, $\sigma_{t}[34]$ :

$$
V(z)=\frac{1}{2} \operatorname{erfc}\left(\frac{z-\langle t\rangle}{\sqrt{2} \sigma_{t}}\right)+\frac{\sigma_{t}}{\sqrt{2 \pi}} \exp \left[-\frac{1}{2}\left(\frac{z-\langle t\rangle}{\sigma_{t}}\right)^{2}\right]
$$

In writing down the previous equation we implicitly assumed a normal (Gaussian) thickness distribution function, which is in general well suited to describe roughness in thin films, especially for films exhibiting a rather low roughness. $G(z)$ is the correlation function of the crystal deformation [25] that describes the effect of heterogeneous strain, i.e. random fluctuations of the strain, $\delta e_{z z}$, around its average value $e_{z z}$ (analysed in the previous section). The exact expression of $G(z)$ depends on the nature of the defects responsible for the heterogeneous strain. When these defects are a priori unknown, as is the case here, it is then useful to have recourse to a phenomenological description of the state of strain [35]. In such a 
description we assume $\delta e_{z z}$ to obey some particular probability distribution function. For instance, in the case of a centred symmetrical Lévy-stable distribution function, we obtain [35]:

$$
G(z)=\exp \left(-\frac{1}{2}\left|h_{z} z\right|^{\gamma} \varepsilon_{z z}^{\gamma}\right)
$$

where $\gamma \in(0,2]$ is the tail index of the distribution [36] (for instance, $\gamma=2$ for a Gaussian distribution, $\gamma=1$ for a Lorentzian distribution). We make use of the versatile Lévy-stable distribution in order to compensate for the limitations of the Gaussian distribution which is known to fail to accurately describe the actual shape of experimental diffraction profiles (see Ref. 35 and references therein). $\varepsilon_{z z}$ is the 'characteristic width' of the distribution [35] of $\delta e_{z z}$. In the analysis of the $Q_{z}$-scans it turned out that the best results were obtained with $\gamma=2$, so that $\varepsilon_{z z}$ is simply the standard deviation of the probability distribution function, that is $\varepsilon_{z z}=<\delta e_{z z}^{2}>^{1 / 2}$. Finally, $R(z)$ is the Fourier transform of the resolution function of the diffractometer which is very well described by a Gaussian function in most scanning geometries [19].

Equations (1)-(3) have been used to simulate the experimental $Q_{z}$-scans using a leastsquares fitting procedure. Since morphological effects (equation 2) are independent of $h_{z}$, whereas heterogeneous strain effects (equation 3) scale with $h_{z}$, the analysis of several $(00 l)$ $Q_{z}$-scans enables one to clearly distinguish between morphology-induced $\left(<t>\right.$ and $\sigma_{t}$ ) and strain-induced $\left(\varepsilon_{z z}\right)$ features. Figure 4a shows that equation (1) fits the data remarkably well over almost 4 orders of magnitude of intensity, with only 3 fitting parameters $\left(<t>, \sigma_{t}\right.$, and $\varepsilon_{z z}$ ). Moreover, it turned out that the heterogeneous strain is vanishingly small, $\varepsilon_{z z}=6.5 \times 10^{-6}$. In other words, the heterogeneous strain in the direction perpendicular to the surface is close to zero, which indicates that the film is almost free of defects. This result fits very well in the framework of the domain-matching epitaxial growth. The thickness has been estimated to be $140 \mathrm{~nm}$, in excellent agreement with the value (135 nm) obtained by m-line spectroscopy (see next section). The determination of the roughness is less obvious, since this parameter affects the thickness-fringes contrast [34], which is itself dependent on other parameters such as the resolution of the diffractometer for instance. In the present case we estimated $\sigma_{t}$ to be 1-3 nm (i.e. identical fitting qualities were obtained for this range of values). This range of values is in good agreement with the value obtained by atomic force microscopy : $1.7 \mathrm{~nm}$. 
Let us now consider the $Q_{x}$-scans (figure $4 \mathrm{~b}$ ). We first write down the expression of the diffracted intensity in the direction parallel to the surface, for a symmetrical $(00 l)$ reflection with reciprocal lattice vector $\left(0,0, h_{z}\right)[33]$ :

$$
I\left(q_{x}\right)=\int d x \cdot \tilde{R}(x) V(x) G(x) \exp \left(i q_{x} x\right)
$$

where $q_{x}$ is the deviation (in the $x$ direction) of the scattering vector from the Bragg position. We will show below that in the in-plane direction the crystallites building up the film are much larger than in the out-of-plane direction. Consequently, in the present case, the $Q_{x}$-scans are much less sensitive to the crystallite size (and size fluctuation) than the $Q_{z}$-scans, so that an accurate determination of the in-plane crystallite size and shape is not conceivable. The choice of a particular crystallite shape and size distribution is therefore unimportant. In the following we shall consider crystallites having the shape of parallelepipeds (the dimensions of which are distributed according to a lognormal distribution) with average edge length $\langle D>$ and thickness $<t>$, as detailed previously. The corresponding expression of $V(x)$ can be found in Ref. 34. The correlation function $G(x)$ is similar to $G(z)$ [35]:

$$
G(x)=\exp \left(-\frac{1}{2}\left|h_{z} x\right|^{\gamma} \varepsilon_{x z}^{\gamma}\right)
$$

The main difference with equation (4) is that the previous equation involves the off-diagonal component of the heterogeneous strain tensor $\varepsilon_{x z}$, which corresponds to the characteristic width of the probability distribution function of $\delta e_{x z}\left(\delta e_{x z}\right.$ being the angular deviation from the perfect $(001)_{\mathrm{LN}} \|(001)_{\mathrm{A} 1203}$ orientation). $\boldsymbol{\varepsilon}_{x z}$ hence simply corresponds to the mosaicity. A detailed inspection of the (006) $Q_{x}$-scan (figure $4 \mathrm{~b}$ ) reveals that it is actually made of two intensity components: a very narrow coherent (Bragg) peak (indicated by the vertical arrow) and a broad diffuse scattering peak (indicated by the inclined arrow). Such a behavior is now quite often observed in thin films (see Ref. 35 and references therein) and can be attributed to the existence of confined strain fields [35, 37]. To account for the spatial confinement of strain it is necessary to introduce an additional parameter: the correlation length $\xi$. For length scales smaller than $\xi$, the rotations of the lattice planes are correlated giving rise to a mosaicity $\varepsilon_{x z}=\varepsilon_{0}$, whereas for length scales larger than $\xi$, the rotations of the lattice planes are uncorrelated so that the mosaicity asymptotically drops to 0 . Such a behavior can be described with the following equation [35]:

$$
\varepsilon_{x z}(x)=\frac{\varepsilon_{0} \xi}{x}\left[1-\exp \left(-\frac{x}{\xi}\right)\right]
$$


Equations (4)-(6) have been used to simulate the experimental $Q_{x}$-scans. As in the previous case, it can be observed that the model nicely fits the data over 4 orders of magnitude of intensity with only 4 fitting parameters $\left(<D>, \varepsilon_{0}, \xi\right.$ and $\left.\gamma\right)$. The tail-index of the probability distribution function converged to 1.4. This value is intermediate between a Gaussian $(\gamma=2)$ and a Lorentzian $(\gamma=1)$. The most interesting result is that the mosaicity is found to be as low as $0.046^{\circ}$, which is one of the lowest values reported in the literature $\left(0.04^{\circ}\right.$ has been obtained in Ref. 5 ; typical values range between $0.1^{\circ}$ and $0.8^{\circ}$ in this system $[9,11,38]$ ) and hence indicates a high crystalline quality of the film. The lateral dimension of the crystallites is estimated to be $\langle D>\approx 500 \mathrm{~nm}$ which is a rather large value as compared to the literature $[3,5]$. Finally, the simulation revealed that rotations of the lattices planes don't extend throughout the film, but are confined in regions with diameter $\xi=30 \mathrm{~nm}$. These features (high crystalline quality, confinement of the lattice distortions) are in good agreement with the proposed domain-matching epitaxial growth mechanism.

\section{Waveguide characterizations}

The characterizations of the optical guiding properties have been performed using the experimental set-up described earlier and presented in figure 1. Since the wavelength is $633 \mathrm{~nm}$, the low thickness of the film $(<150 \mathrm{~nm})$ allows the propagation of only one mode per polarization. The measurements of the effective indices of the $\mathrm{TM}_{0}$ and $\mathrm{TE}_{0}$ modes performed by the m-line method yields a film thickness of $135 \pm 5 \mathrm{~nm}$ (assuming that the ordinary and extraordinary indices of the $\mathrm{LiNbO}_{3}$ film are equal to those of a bulk single crystal). This value is in good agreement with the thickness obtained by XRD (140 nm, see previous section).

We have assessed the quality of the light propagation in the $\mathrm{LiNbO}_{3}$ film by two different ways. Firstly, we have observed the edge of the substrate (figure 5a). This figure shows that the beam power is confined in the $\mathrm{LiNbO}_{3}$ film. Secondly, the propagation was achieved along the film allowing the evaluation of the propagation losses. The scattered optical power along the propagation axis in the film is recorded by a CCD camera (an example is given in figure $5 \mathrm{~b}$ ). The intensity profile is extracted from the image and processed as outlined in section 2. The measurements are disturbed by the presence of microscopic dust particles on the surface of film that act as additional scattering centres along the mode 
propagation (clearly visible on figure 5b). Such a phenomenon has already been reported [38] and explained by the presence of an electric field created by the spontaneous polarization of the $\mathrm{LiNbO}_{3}$ film. Because of the presence of these particles and the rather low losses in the film, a precise estimation of the propagation losses is almost impossible. A rough estimation in the $\mathrm{TM}_{0}$ mode yields a value of $1.5 \mathrm{~dB} / \mathrm{cm}$ (which is of the same order of magnitude than previously published values $[2,5,38])$. The accuracy is estimated to $\sim 0.5 \mathrm{~dB} / \mathrm{cm}$.

\section{Discussion}

In thin films, optical losses are sensitive both to surface roughness (surface optical losses) and to crystal structure and microstructure (volume optical losses) [40]. Both induce spatial random perturbations of the local effective index $n_{\text {eff }}$ of the guided mode along the propagation axis leading to optical power coupling towards the radiative modes (i.e. not guided). The efficiency of such a coupling and the induced losses are directly linked to the standard deviation value $\sigma$ and the correlation length $L_{C}$ of the random variation of $n_{\text {eff }}$ along the waveguide $[41,42]$. As the waveguide presents a high refractive index contrast and a very low thickness, the guided mode propagates near the cut-off, thus enhancing the effects of any kind of perturbation [39] Considering hemispherical crystallites, Lee et al have shown that the surface roughness tends to scale with the in-plane crystallite size [5].

Therefore, in order to limit the surface roughness, and hence the optical losses, it has been suggested to keep the in-plane crystallite size as low as possible [3,5]. A rule of thumb is that optical losses $<1 \mathrm{~dB} / \mathrm{cm}$ can only be reached with a surface roughness $<1 \mathrm{~nm}$ [9]. An obvious drawback of this approach is that small crystals give rise to a large grain boundary surface area. Since defects (e.g. nanocracks, microcracks...) appear preferentially at grain boundaries [15], a possible way to improve the overall guiding properties of the films is to limit the number of grain boundaries (i.e. favoring in-plane crystal growth) while keeping a low roughness. Moreover, the dimensional properties of the waveguide perturbation (surface and volume) must be taken into account in the evaluation. The optical losses are indeed maximized when $L_{C}$ is very close to the quarter of the wavelength in the waveguide [41-43] which corresponds in our case to $80 \mathrm{~nm}$ (taking into account the refractive index of the film).

We have shown in this work that it is possible to grow high quality LN films with a rather low roughness $(1.7 \mathrm{~nm})$ and large in-plane crystallite size $(\sim 500 \mathrm{~nm})$. The lateral 
growth of the crystals has been favored by avoiding the appearance of unwanted secondary phases or secondary orientations. The analysis of XRD data indeed revealed that the film composition $\left(x_{\mathrm{Li}}=48.7 \%\right)$ is very close to the composition of the target, the film is free of Lideficient phases, and the amount of $60^{\circ}$ variant is as low as $6 \%$. In the direction normal to the surface the film is single-crystalline (i.e. there is one crystal in the film thickness) and free of structural defects. The high crystalline quality of the film is further attested by the low mosaicity (only $0.046^{\circ}$ ), that is to say that the large crystalline domains (500 nm wide and 140 $\mathrm{nm}$ thick) building up the film are only weakly disoriented with respect to each other which is undoubtedly a favorable situation for light propagation. The analysis of the state of strain revealed that the film is free of epitaxial strain, as a result of the domain-matching epitaxial growth mechanism, but it is under high thermal stress as a result of the difference in coefficient of thermal expansion between the film and the substrate. It is particularly important to avoid thermal strain relaxation, since thermal strain relaxation is mainly driven by the formation of cracks which is obviously detrimental for the guiding properties.

This point can be illustrated by the analysis of a sample grown under different conditions (sample 2, $P_{\mathrm{O} 2}=0.9 \mathrm{mbar}$ and $F=1.8 \mathrm{~J} / \mathrm{cm}^{2}$ ) and exhibiting similar structural properties. The corresponding (006) and (018) RSMs, $Q_{z^{-}}$and $Q_{x}$-scans are given in figure 6 and 9. The crystallographic orientation of the film is identical to the previous case with an amount of $60^{\circ}$ variant evaluated to $5 \%$. The film thickness, determined from the analysis of the $Q_{z}$-scans, is $135 \mathrm{~nm}$ (with a roughness estimated at 1-3 nm as in the previous case), the $\mathrm{Li}$ molar fraction, determined from the analysis of the (018) map, is $x_{\mathrm{Li}}=48.2 \%$ and no Lideficient phases could be detected. Despite these similarities, no propagation could be observed in sample 2 while an m-line could be clearly observed. The coupling area where the incoming laser beam is injected into the film through the prism is the place of a very important light scattering. This indicates that the optical power is initially coupled to the film (put into evidence by the visualization of an m-line) and is immediately extracted by a scattering process. We suggest that this difference in the guiding behavior can be explained by the different in-plane crystallite size between the samples as well as by the different state of strain. The analysis of the $Q_{x}$-scans of sample 2 indeed revealed that the in-plane crystallite size is 10 times lower than in the previous sample, i.e. we obtained $\langle D>=50 \mathrm{~nm}$ associated with a mosaicity more than 2 times higher $\left(\varepsilon_{0}=0.1^{\circ}\right)$. In contrast with sample 1 , the in-plane 
crystallite size of sample 2 is very close to the quarter-wavelength value $(80 \mathrm{~nm})$ so that high optical losses can be expected in this film.

Together with this lowering of the in-plane crystallite size we also observe the onset of thermal strain relaxation. The analysis of the (018) RSM reveals that the residual strain in the film is $0.47 \%$ which corresponds to a $20.5 \%$ thermal strain relaxation (i.e. $\left(e_{\text {th }}-e_{x x}\right) / e_{\text {th }}=$ $20.5 \%$ ) so that cracks are likely to appear in this sample, which is another possible reason for the lack of waveguiding in this sample. The reduced crystallite size, associated with the corresponding increase of the grain boundary surface, is undoubtedly at the origin of the thermal strain relaxation [15].

We can also notice that this thermal strain relaxation occurs inhomogeneously throughout the film thickness. The $Q_{z}$-scans are indeed asymmetrical and broadened towards low $Q_{z}$ values (i.e. higher $c$ values, this is indicated by the arrows in figure 7) which indicates a variation of the lattice parameters across the film thickness [44,45]. This is further confirmed by the (018) RSM (figure 6) where the reciprocal lattice point corresponding to the film is clearly broadened along the relaxation line [46] (indicated by the dashed line), that is the line joining the position of the reflections corresponding to the fully-strained and strainrelaxed film.

\section{Conclusions}

High-quality LN epitaxial films have been grown onto (001) sapphire substrates by pulsed laser deposition. The structural and nanostructural properties of the films have been investigated by high-resolution XRD, especially using the reciprocal space mapping technique. The film composition as well as the amount of strain, the film thickness, the film roughness, the lateral extension of the crystallites and the mosaicity have been determined thanks to this technique, associated with simulations of the diffracted intensity distribution in reciprocal space. Waveguiding properties of the films have been investigated using the prism coupling method. This technique presents the advantages to be non-destructive, simple to implement (no technological process needed) and suitable for optical power coupling in very low thickness planar waveguides. Optical propagation has been clearly observed in thin films grown under optimized conditions. We have shown that single crystalline-like films (i.e. exhibiting a large in-plane crystallite size, a low mosaicity, no impurities and weak 
roughness) with low optical losses can be grown by PLD. Besides, we have also shown that the existence of mosaic structure in the film (i.e. nanometric in-plane crystallite size and increased mosaicity) is detrimental to the waveguiding properties.

Such LN waveguides are candidates to be the active layer in complex integrated optics components for electro-optical or non-linear applications. It has to be emphasized that, because of the large difference in the refractive indices of LN and sapphire, single-mode waveguiding can only be achieved in relatively thin films $(<300 \mathrm{~nm})$. This low thickness makes them advantageous and may lead to enhanced performances in both domains either by allowing lower electrode distance or by increasing the optical power density in the film, respectively. However, this work has now to be followed by the development of many technological processes whose main aim will be to ensure lower loss and increased compactness of the optical power coupling with the waveguide (injection and extraction). For that purpose, many solutions are available among the usual integrated optics and optoelectronics technologies (e.g. for InP based devices - another kind of very high index difference optical waveguides). Thus, tapered waveguides or directional coupling with an additional layer of higher dimensions, both to match the mode field diameter of optical fibers might be applicable to the LN films. 


\section{References}

[1] Arizmendi L 2004 Phys. Stat. Sol. (a) 201253

[2] Takahashi M, Yamauchi K, Yagi T, Nishiwaki A, Wakita K, Ohnishi N, Hotta K, Sahashi I 2004 Thin Sol. Films 458108

[3] Dogheche E, Lansiaux X, Remiens D 2003 J. Appl. Phys. 931165

[4] Callejo D, Manotas D, Serrano M D, Bermudez V, Agullo-Rueda F, Dieguez E $2001 \mathrm{~J}$. Cryst. Growth 226448

[5] Lee S Y, Feigelson R S 1998 J. Cryst. Growth 186594

[6] Aubert P, Garry G, Bisano R, Olivier J, Garcia-Lopez J, Urlacher C 1995 Microelectronic Eng. 29107

[7] Nakata Y, Gunji S, Okada T, Maeda M, Fotakis C 2004 Appl. Phys. A 791279

[8] Kim D W, Lee S H, Noh T W 1998 Mater. Sci. Eng. B 56251

[9] Derouin T A, Lakeman C D E, Wu X H, Speck J S, Lange F F 1997 J. Mater. Res. 12 1391

[10] Roemer A, Millon E, Vincent B, Boudrioua A, Pons-Y-Moll O, Defourneau R M, Seiler W 2004 J. Appl. Phys. 953041

[11] Balestrino G, Martellucci S, Medaglia P G, Paoletti A, Petrocelli G, Tebano A, Tucciarone A, Gelli F, Giorgetti E, Sottini S, Tapfer L 2001 Appl. Phys. Lett. 781204

[12] Kakehi Y, Okamoto A, Sakurai Y, Nishikawa Y, Yotsuya T, Ogawa S 2001 Appl. Surf. Sci. 169560

[13] Bouquet V, Leite E R, Longo E, Varela J A, Guilloux Viry M, Perrin A 2001 J. Eur. Ceram. Soc. 211521

[14] Vasconselos N S L S, Vasconselos J S, Bouquet V, Zanetti S M, Leite E R, Longo E, Soledade L E B, Pontes F M, Guilloux Viry M, Perrin A, Bernardi M I, Varela J A 2003 Thin Sol. Films 436213

[15] Veignant F, Gandais M, Aubert P, Garry G 1999 J. Cryst. Growth 196141

[16] Terabe K, Gruverman A, Matsui Y, Iyi N, Kitamura K 1996 J. Mater. Res. 113152

[17] Box G E P, Wilson K B 1951 J. Royal Stat. Soc. B 131

[18] Boulle A, Masson O, Guinebretière R, Dauger A 2001 Appl. Surf. Sci. 180322 
[19] Boulle A, Masson O, Guinebretière R, Lecomte A, Dauger A 2002 J. Appl. Crystallogr. 35606

[20] Masson O, Boulle A, Guinebretière R, Lecomte A, Dauger A 2005 Rev. Sci. Instr. 76 063912

[21] Ulrich R, Torge R 1973 Appl. Opt. 122901

[22] Trampert A, Ploog K H 2000 Cryst. Res. Technol. 35793

[23] Narayan J, Larson B C 2003 J. Appl. Phys. 93278

[24] Zheleka T, Jagannadham K, Narayan J 1994 J. Appl. Phys. 75860

[25] Pietsch U, Holý V, Baumbach T 2004 High-resolution X-ray scattering - From thin films to lateral nanostructures (Springer, Berlin).

[26] Veignant F, Gandais M, Aubert P, Garry G 1998 Thin Sol. Films 336163

[27] Hirthe J P, Lothe J 1992 Theory of dislocations (Krieger Publishing compagny, Malabar Florida).

[28] Conchon F, Boulle A, Guinebretière R, Dooryhée E, Hodeau J -L, Girardot C , Pignard S, Kreisel J, Weiss F, Libralesso L, Lee T. L. 2008 J. Appl. Phys. 103, 123501

[29] Conchon F, Boulle A, Guinebretière R, Girardot C, Pignard S, Kreisel J, Weiss F, Dooryhée E, Hodeau J -L 2007 Appl. Phys. Lett. 91192210

[30] Malovichko G, Cerclier O, Estienne J, Grachev V, Kokanyan E, Boulesteix C 1995 J. Phys. Chem. Solids 561285

[31] Schuster M, Gervais P O, Jobst B, Hösler W, Averbeck R, Riechert H, Iberl A, Stömmer R 1999 J. Phys. D: Appl. Phys. 32 A56

[32] Kilburger S Réalisation et caractérisation d'hétérostructures à base de couches minces de $\mathrm{LiNbO}_{3}$ pour des applications en optique intégrée $2008 \mathrm{Ph}$. D. Thesis, Université de Limoges, France.

[33] Boulle A, Guinebretière R, Masson O, Bachelet R, Conchon F, Dauger A 2006 Appl. Surf. Sci. 25395

[34] Boulle A, Conchon F, Guinebretière R 2006 Acta. Crystallogr. A 6211

[35] Boulle A, Guinebretière R, Dauger A 2005 J. Phys. D: Appl. Phys. 383907 
[36] Nolan J P 1998 Stat. Probabil. Lett. 38187

[37] Boulle A, Guinebretière R, Dauger A 2005 J. Appl. Phys. 97073503

[38] Lansiaux X, Dogheche E, Remiens D, Guilloux-Viry M, Perrin A, Ruterana P $2001 J$. Appl. Phys. 905274

[39] Sterligov V A, Lysenko S I, Aschieri P, Baldi P, Chanvillard L, De Micheli M 2002 Appl. Opt. 411418

[40] Aubert P 1996 Croissance épitaxiale par ablation laser de niobate de lithium sur saphir : Structure crystalline et propriétés optiques, Ph. D. Thesis, Université Pierre et Marie Curie, Paris, France

[41] Lee K K, Lim D R, Luan H, Agarwal A, Foresi J, Kimerling L C 2000 Appl. Phys. Lett. 771617

[42] Payne F P, Lacey J P R 2000 Opt. Quantum Electron. 26977

[43] Haus H 1984 Waves and Fields in Optoelectronics (Prentice-Hall, Englewood Cliffs, NJ).

[44] Boulle A, Masson O, Guinebretière R, Dauger A 2003 J. Appl. Crystallogr. 361424

[45] Boulle A, Conchon F, Guinebretière R 2009 J. Appl. Crystallogr. 42 in press.

[46] Heinke H, Kirchner V, Selke H, Chierchia R, Ebel R, Einfeldt S, Hommel D $2001 \mathrm{~J}$. Phys. D: Appl. Phys. 34, A25 


\section{Figure Caption}

Figure 1: Prism-based injection set-up for injection and loss measurements of the guided mode.

Figure 2: reciprocal space maps of the (006) (a) and (018) (b) reflections of the $\mathrm{LiNbO}_{3} / \mathrm{Al}_{2} \mathrm{O}_{3}$ system. The dashed line is the relaxation line, joining the (018) reflections corresponding to the fully strained and strain-relaxed films $\left(\right.$ scale bar $\left.=0.05 \AA^{-1}\right)$.

Figure 3: $\phi$-scan recorded from the (018) reflection of $\mathrm{LiNbO}_{3}$. The (018) reflection of $\mathrm{Al}_{2} \mathrm{O}_{3}$ appears at $\phi=0^{\circ}$. Inset : schematic representation of the $\mathrm{LiNbO}_{3} / \mathrm{Al}_{2} \mathrm{O}_{3}$ structure for the main orientation (a) and for the $60^{\circ}$ epitaxial variant (b) (the large spheres are the oxygen ions and the interface is symbolized by the horizontal dashed line).

Figure 4: (a) $Q_{z}$-scans of the (006) and (00 12) reflections (black circles : experimental data ; red curve : simulation). (b) $Q_{x}$-scans of the (006) and (00 12) reflections (black circles : experimental data ; red curve : simulation). The vertical arrow indicates the coherent peak ; the inclined arrow indicates the broad diffuse peak.

Figure 5: (a) View of the propagation streak within a $\mathrm{LiNbO}_{3}$ waveguide (scale bar $=1 \mathrm{~mm}$ ). (b) View of the edge of the substrate.

Figure 6: reciprocal space maps of the (006) and (018) reflections of the $\mathrm{LiNbO}_{3} / \mathrm{Al}_{2} \mathrm{O}_{3}$ system (sample 2). The dashed line is the relaxation line, joining the (018) reflections corresponding to the fully strained and strain-relaxed films (scale bar $=0.05 \AA^{-1}$ ).

Figure 7: (a) $Q_{z}$-scans of the (006) and (00 12) reflections of sample 2 (black circles : experimental data ; red curve : simulation). The arrows indicate the peak asymmetry. (b) $Q_{x^{-}}$ scans of the (006) and $(00$ 12) reflections of sample 2 (black circles : experimental data ; red curve : simulation). 
Figure 1

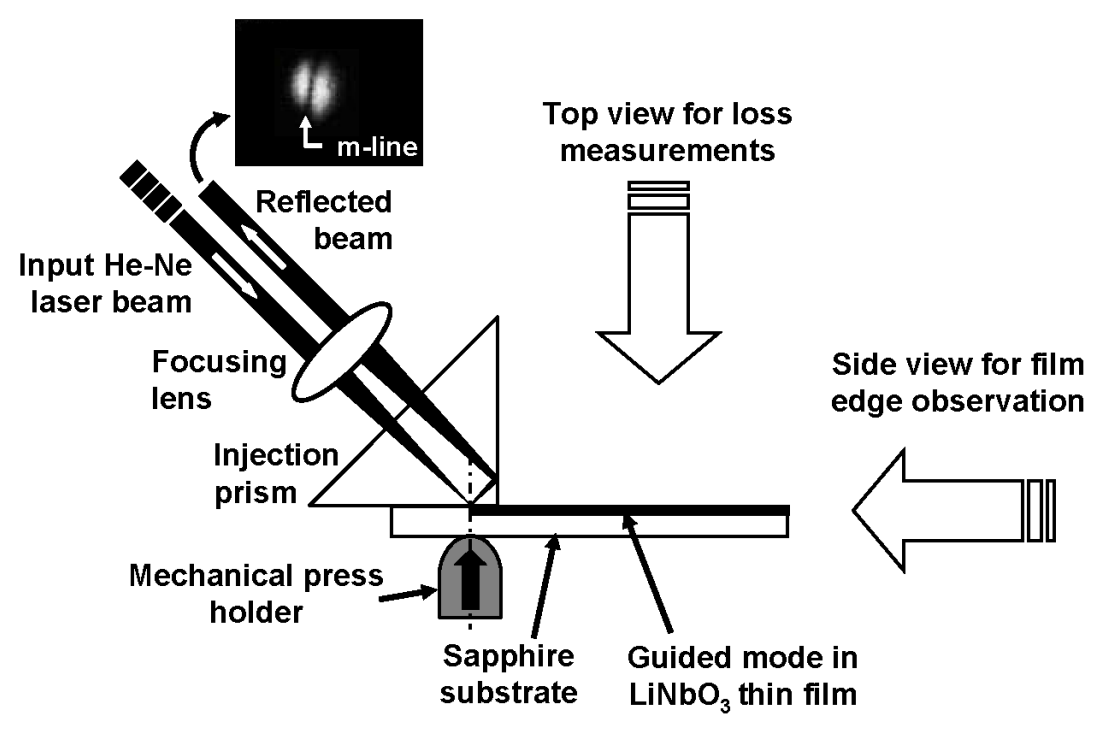


Figure 2

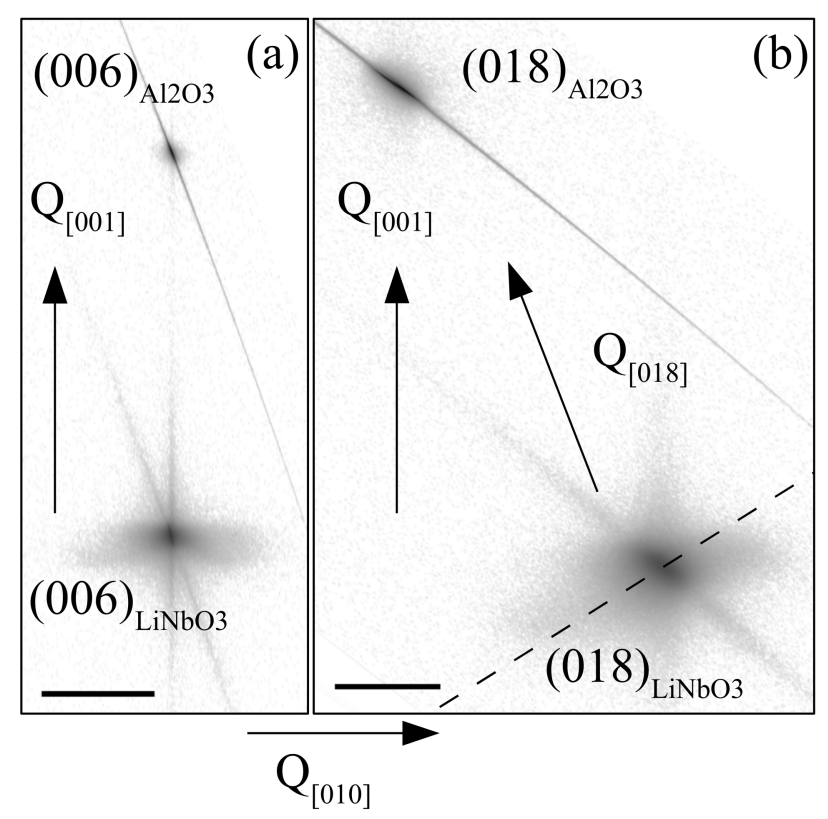


Figure 3

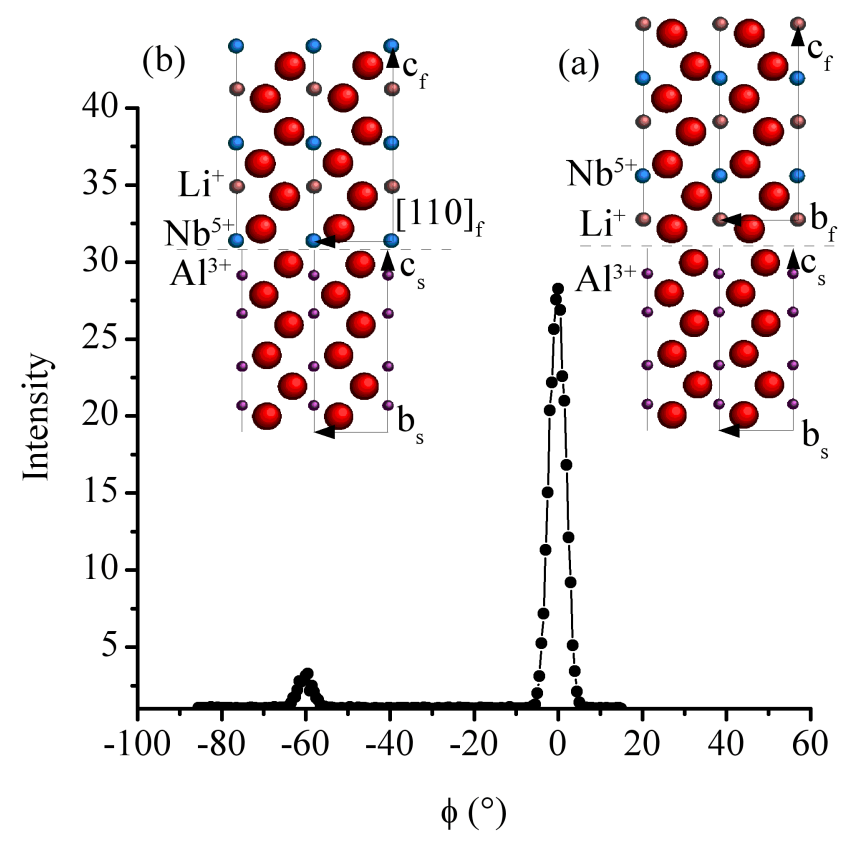


Figure 4

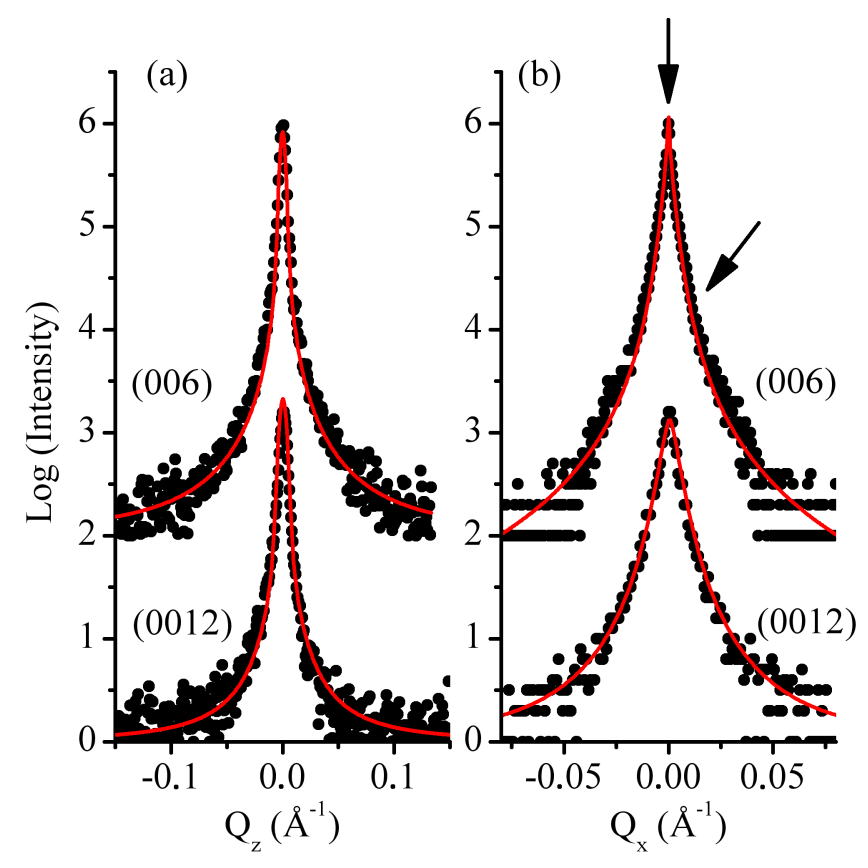


Figure 5
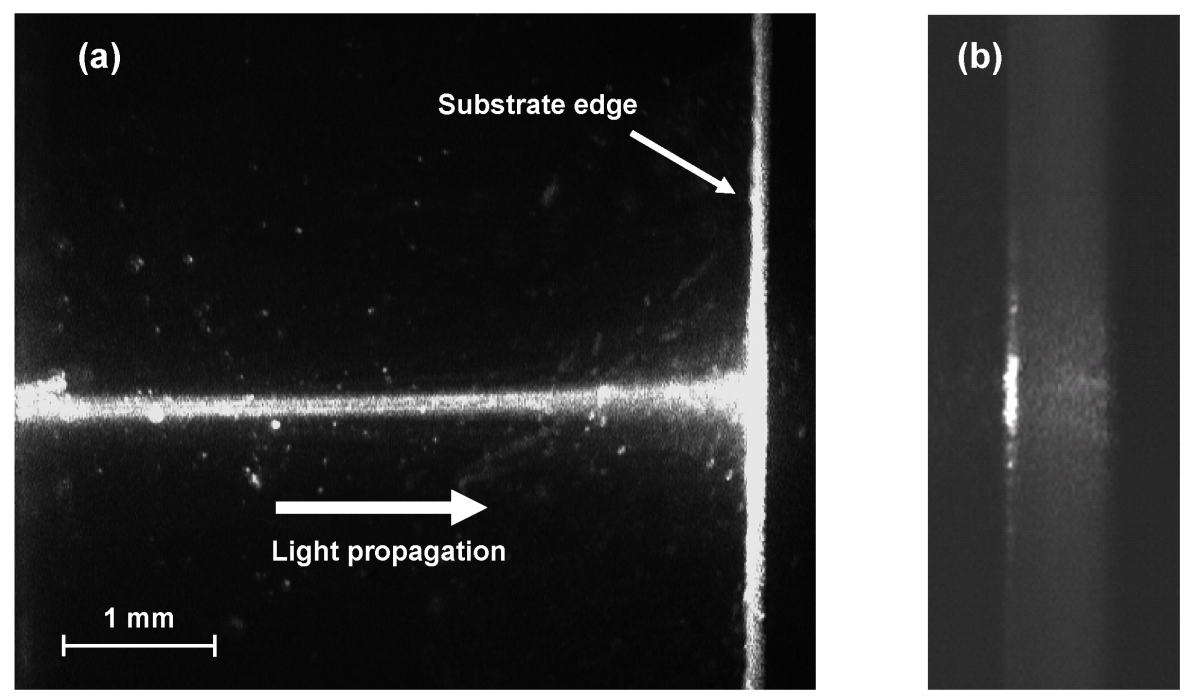
Figure 6

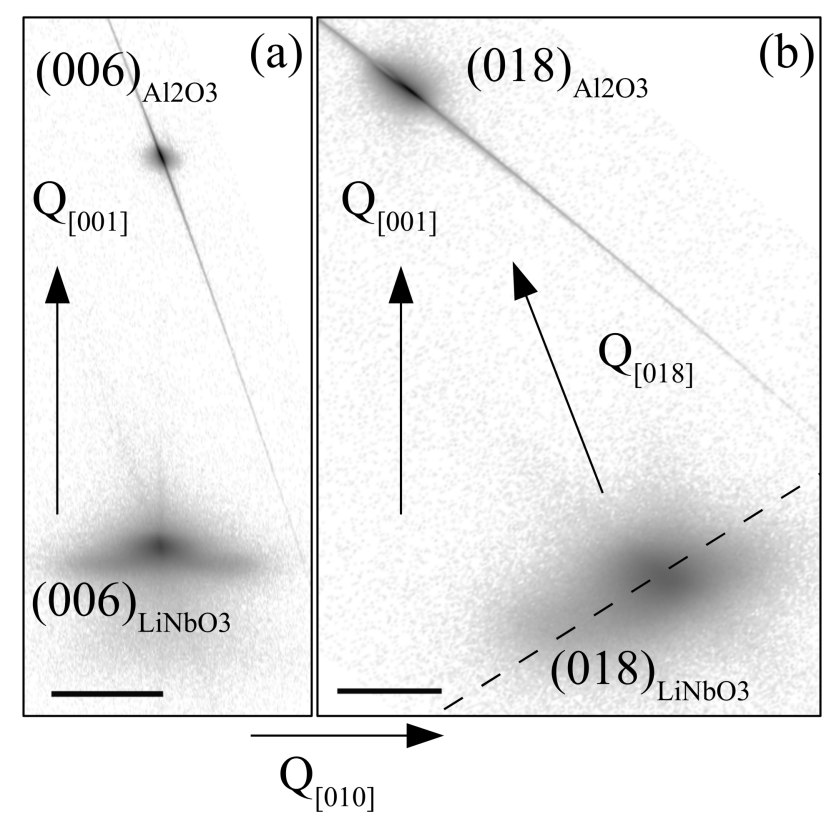


Figure 7

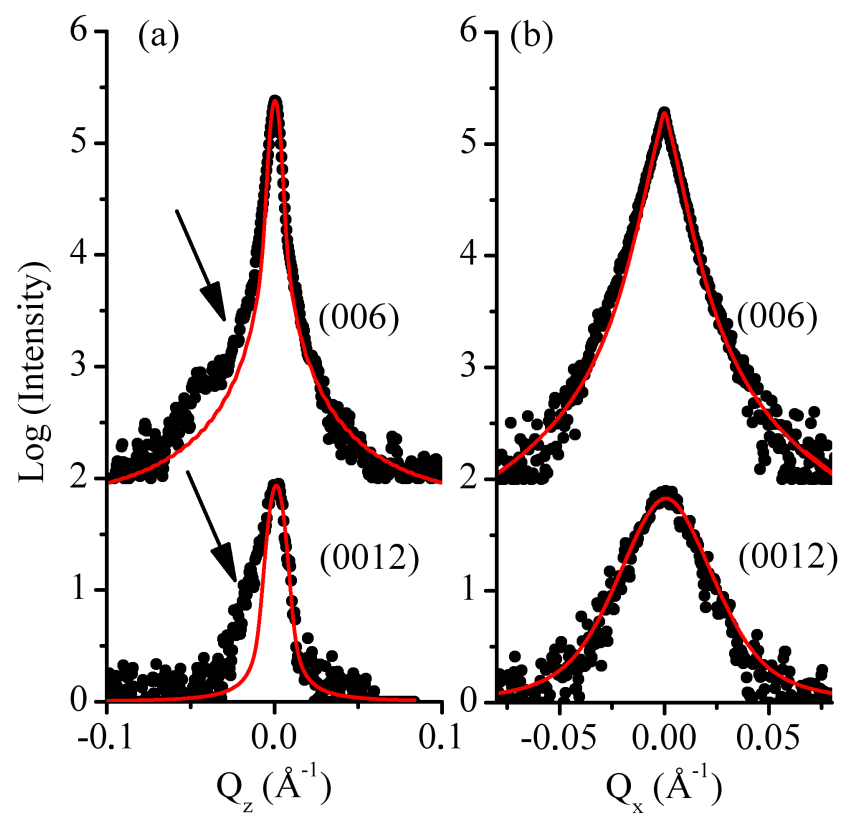

